\title{
Erfahrungen mit trägerübergreifenden IQM-Peer-Review-Verfahren
}

\section{O. Rink}

Qualitätsmanagement

Schlüsselvörter

- Peer Review

Fehlerkultur

Daktives

Qualitätsmanagement

kontinuierlicher

Verbesserungsprozess

Keywords

peer review

- medical error handling

active quality management

continuous quality improvement
Institut

Initiative Qualitätsmedizin IQM

Berlin

Bibliografie

DOI $10.1055 / \mathrm{s}-0031-1286081$

Dtsch Med Wochenschr 2011;

136: S52 - (c) Georg Thieme

Verlag KG Stuttgart · New York

ISSN 0012-0472

\section{Korrespondenz}

\section{Dr. med. Oda Rin}

Initiative Qualitätsmedizin $\mathrm{IQ}^{\mathrm{M}}$

Leiterin Fachausschuss

Peer Review

Friedrichstrasse 166

10117 Berlin

Tel. $0173 / 6078714$

eMail

oda.rink@helios-kliniken.de

\author{
IQM-Peer-Review
}

Seit 2009 werden bei IQM (Initiative Qualitätsmedizin) trägerübergreifende Peer-Review-Verfahren durchgeführt. Nach vier Pilotprojekten im Jahr 2009 wurden 2010 anschließend 21 und 2011 weitere 44 Verfahren in allen Trägergruppen organisiert. Analysiert wurden zunächst internistische und neurologische sowie chirurgische und unfallchirurgische/orthopädische Krankheitsbilder, zusätzlich komplexe Erkrankungen mit Langzeitbeatmungen.

Zusammenfassung der Ergebnisse:

1. Durch das Kennzahlensystem kann Optimierungspotenzial sehr gut identifiziert werden, in 2010 lag es bei $64 \%$ (Spanne 20 bis $90 \%$ );

2. in $67 \%$ der Kliniken war die Dokumentation nicht befriedigend oder ungenügend:

3. in 57\% der Kliniken wurde der Behandlungsprozess nicht ausreichend hinterfragt;

4. in $52 \%$ der Kliniken wurde die Behandlung nicht inhaltlich adäquat oder nicht zeitgerecht durchgeführt.

Der Handlungsbedarf im Einzelnen wird gemeinsam in einer kollegialen Diskussion erarbeitet und im Protokoll konkret beschrieben. Die Umsetzung der Ergebnisse obliegt der Klinik vor Ort und wird in der Kontrolle der Kennzahlen sichtbar.

\section{Erfahrungen \\ $\nabla$}

In der organisatorischen Durchführung des Verfahrens sind die Hauptprobleme die Zusammenstellung der Review-Teams und die Terminplanung der Reviews.

Die Akzeptanz des ärztlich gesteuerten Verfahrens wird vorwiegend durch die kollegiale Diskussion aller Fälle auf Augenhöhe erreicht. Dies muss auch in der Zusammenstellung der interdisziplinären und trägerübergreifenden Teams berücksichtigt werden und hat zu einer intensivierten Ausbildung der Peers nach dem Curriculum der Bundesärztekammer (BÄK) geführt.

Gegenseitiges voneinander Lernen ist das Grundprinzip der Reviews. Es wird perspektivisch jeder Peer selbst in seiner Klinik „gereviewt“, um die Erfahrung mit den unterschiedlichen Rollen und Situationen im Review-Verfahren zu intensivieren.
Die Optimierung interdisziplinärer Prozesse und die offene Diskussion von Verbesserungspotenzialen führen zu einer offenen Fehlerkultur. [1]

Die Einbindung der Geschäftsführung ist sowohl zur Durchsetzung der Ergebnisse als auch zur Beschleunigung von Veränderungen notwendig und befördert den kontinuierlichen Verbesserungsprozess. Dieses aktive Qualitätsmanagement wird von den Klinikleitungen als entscheidender Vorteil für die Positionierung der Krankenhäuser in der Zukunft gesehen.

Autorenerklärung: Es bestehen keine Interessenkonflikte, weder finanzieller noch sonstiger Art.

\section{Literatur}

1 Rink O, Eberlein-Gonska M. Jahrbuch Qualitätsmedizin 2010. Peer Review - wie wir Qualität verbessern lernen. Berlin: 2010: 59-69 